\title{
A Goursat decomposition for polyharmonic functions in Euclidean space
}

\author{
Fred Brackx, Richard Delanghe, Hennie De \\ Schepper and Vladimir Souček
}

\begin{abstract}
The Goursat representation formula in the complex plane, expressing a real-valued biharmonic function in terms of two holomorphic functions and their anti-holomorphic complex conjugates, is generalized to Euclidean space, expressing a real-valued polyharmonic function of order $p$ in terms of $p$ so-called monogenic functions of Clifford analysis.
\end{abstract}

Mathematics Subject Classification (2010). 30G35.

Keywords. Goursat decomposition, polyharmonic functions.

This paper is dedicated to the memory of Professor J. Keller

\section{Introduction}

In classical complex analysis it is well-known that harmonic functions and holomorphic functions are intimately related. If $\Omega$ is a simply connected region in the complex plane and $u(x, y)$ is a real-valued harmonic function in $\Omega$, then there exists a second real-valued harmonic function $v(x, y)$, called a harmonic conjugate to $u$ in $\Omega$, such that $f(z)=u(x, y)+i v(x, y)$ is holomorphic in $\Omega$, i.e. a null solution of the Cauchy-Riemann operator $\partial_{\bar{z}}=\frac{1}{2}\left(\partial_{x}+i \partial_{y}\right)$. It follows that $u(x, y)=\frac{1}{2}(f(z)+\bar{f}(z))=\operatorname{Re} f(z)$, in other words: any realvalued harmonic function in $\Omega$ can be decomposed as a sum of a holomorphic function and its complex conjugate, the latter being anti-holomorphic, i.e. a null solution of the conjugate Cauchy-Riemann operator $\partial_{z}=\frac{1}{2}\left(\partial_{x}-i \partial_{y}\right)$.

Already in 1898 Goursat [10] obtained a representation of a biharmonic function, i.e. a null solution of $\Delta^{2}$, in terms of two holomorphic functions and their anti-holomorphic complex conjugates: if $u(x, y)$ is a real-valued biharmonic function in $\Omega$, still a simply connected region in the complex plane, then there exist holomorphic functions $\varphi(z)$ and $\psi(z)$ in $\Omega$ such that

$$
u(x, y)=\varphi(z)+\bar{\varphi}(z)+\bar{z} \psi(z)+z \bar{\psi}(z)=2 \operatorname{Re}(\varphi(z)+\bar{z} \psi(z))
$$


Note that $\varphi \in \operatorname{Ker} \partial_{\bar{z}}, \bar{\varphi} \in \operatorname{Ker}_{z}, \bar{z} \psi \in \operatorname{Ker} \partial_{\bar{z}}^{2}, z \bar{\psi} \in \operatorname{Ker} \partial_{z}^{2}$. It follows that

$$
u(x, y)=\gamma(x, y)+x \alpha(x, y)+y \beta(x, y)
$$

with $\gamma(x, y)=2 \operatorname{Re} \varphi(z), \alpha(x, y)=2 \operatorname{Re} \psi(z)$ and $\beta(x, y)=2 \operatorname{Im} \psi(z)$, in other words: any real-valued biharmonic function in $\Omega$ may be decomposed in terms of three harmonic functions, two of them being conjugate harmonic.

In [11] and [9] the number of harmonic functions needed for this kind of representation was reduced to two: given a real-valued biharmonic function $u(x, y)$ in $\Omega$, there exist harmonic functions $g_{0}, h_{0}, g_{1}, h_{1}, g_{2}, h_{2}$ such that

$$
u(x, y)=g_{0}(x, y)+\left(x^{2}+y^{2}\right) h_{0}(x, y)
$$

or

$$
u(x, y)=g_{1}(x, y)+x h_{1}(x, y)
$$

or

$$
u(x, y)=g_{2}(x, y)+y h_{2}(x, y)
$$

In fact, (3) is a decomposition of the so-called Almansi type. In [1] Almansi obtained a decomposition in three-dimensional Euclidean space of a realvalued polyharmonic function $U(x)$ satisfying $\Delta^{k} U=0$ in a star domain, in terms of harmonic functions $h_{0}, h_{1}, \ldots, h_{k-1}$ and powers of $|x|^{2}$ :

$$
U(x)=h_{0}(x)+|x|^{2} h_{1}(x)+\ldots+|x|^{2(k-1)} h_{k-1}(x)
$$

In its turn (6) was a generalization to polyharmonic functions of the Gauss decomposition of a polynomial in terms of harmonic polynomials and powers of $|x|^{2}$. This classical result can be expressed by saying that $\left(|x|^{2}, \Delta\right)$ is a Fischer pair for the space of all polynomials; the pair $(P, Q(D))$, consisting of a polynomial and a differential operator, is called a Fischer pair if for each polynomial $p$ there exist unique polynomials $q$ and $r$ such that $Q(D) r=0$ and $p=P q+r$. In [8] Fischer proved that for every homogeneous polynomial $P$, the pair $\left(P(x), P^{*}(D)\right)$ is a Fischer pair, where $P^{*}$ denotes the polynomial obtained from $P$ by conjugation of its coefficients.

The aim of this paper is to generalize, in a first step, the Goursat representation formula for biharmonic functions in the complex plane to polyharmonic functions in the whole of Euclidean space; the case of polyharmonic functions in an open region of Euclidean space is a topic for further research. This, naturally, necessitates a generalization to higher dimension of the notion of holomorphy. For that we can use the framework of Clifford analysis, more in particular the notion of monogenic function, i.e. a null solution of a generalized Cauchy-Riemann operator acting on functions defined in Euclidean space $\mathbb{R}^{m+1}$ and with values in the Clifford algebra $\mathbb{R}_{m+1}$ constructed over $\mathbb{R}^{m+1}$, or subspaces thereof. In this context it should be mentioned that a generalization of the Goursat representation formula to $\mathbb{R}^{3}$ in the framework of quaternionic analysis was obtained in [3], and a similar generalization to $\mathbb{C}^{2}$ in $[4]$. 
The structure of the paper is as follows. In section 2 we briefly recall the basics of Clifford algebra and Clifford analysis. In section 3 we prove some elementary but useful results on polyharmonic functions which will be exploited in the sequel. Section 4 is devoted to the Goursat representation formula for biharmonic functions in Euclidean space. This paves the way for the general case of polyharmonic functions in Euclidean space in Section 5.

\section{Clifford algebra and Clifford analysis: some basics}

Let $\mathbb{R}^{0, m+1}$ be the real vector space $\mathbb{R}^{m+1}(m>1)$ provided with a nondegenerate quadratic form of signature $(0, m+1)$ and let $e=\left(e_{0}, \ldots, e_{m}\right)$ be an orthonormal basis of $\mathbb{R}^{0, m+1}$. Then $e$ generates the universal Clifford algebra $\mathbb{R}_{0, m+1}$ over $\mathbb{R}^{0, m+1}$ and, embedded in $\mathbb{R}_{0, m+1}, \underline{e}=\left(e_{1}, \ldots, e_{m}\right)$ generates the universal Clifford algebra $\mathbb{R}_{0, m}$ over $\mathbb{R}^{0, m}$. The multiplication in the Clifford algebra $\mathbb{R}_{0, m+1}$ is non-commutative; it is governed by the rules

$$
\begin{cases}e_{i}^{2}=-1, & i=0, \ldots, m \\ e_{i} e_{j}+e_{j} e_{i}=0, & i \neq j, i, j=0, \ldots, m\end{cases}
$$

A basis for $\mathbb{R}_{0, m+1}$ is given by $\left(e_{A}\right)_{A \subset\{0, \ldots, m\}}$ where for $A=\left(i_{1}, \ldots, i_{r}\right)$ with $0 \leq i_{1}<i_{2}<\ldots<i_{r} \leq m$, we put $e_{A}=e_{i_{1}} e_{i_{2}} \ldots e_{i_{r}}$, while $e_{\emptyset}=1$ is the identity element. Any $a \in \mathbb{R}_{0, m+1}$ may thus be written as $a=\sum_{A} a_{A} e_{A}$ with $a_{A} \in \mathbb{R}$ or still as $a=\sum_{k=0}^{m+1}[a]_{k}$ where $[a]_{k}=\sum_{|A|=k} a_{A} e_{A}$ is the so-called $k$-vector part of $a(k=0,1, \ldots, m+1)$. If we denote the space of $k$-vectors by $\mathbb{R}_{0, m+1}^{k}$, then the Clifford algebra $\mathbb{R}_{0, m+1}$ decomposes as $\bigoplus_{k=0}^{m+1} \mathbb{R}_{0, m+1}^{k}$. Conjugation in $\mathbb{R}_{0, m+1}$ is defined as the anti-involution $a \rightarrow \bar{a}$ for which $\overline{e_{i}}=-e_{i}, i=0,1, \ldots, m+1$. For the Clifford algebra $\mathbb{R}_{0, m}$ the construction of a basis and the decomposition into subspaces of $k$-vectors $(k=0,1, \ldots, m)$ is completely similar.

The Euclidean spaces $\mathbb{R}^{m}$ and $\mathbb{R}^{m+1}$ are identified with the subspaces of 1 -vectors in the respective Clifford algebras $\mathbb{R}_{0, m}$ and $\mathbb{R}_{0, m+1}$, by putting $\underline{x}=\sum_{j=1}^{m} e_{j} x_{j}$ and $x=\sum_{i=0}^{m} e_{i} x_{i}$. It follows that $x=x_{0} e_{0}+\underline{x}$. For further use we also introduce the new variable

$$
z=\bar{e}_{0} x=x_{0}+\bar{e}_{0} \underline{x}
$$

and its conjugate $\bar{z}=x_{0}-\overline{e_{0}} \underline{x}$. The multiplication of any two vectors $x$ and $y$ is given by

$$
x y=x \circ y+x \wedge y
$$

with

$$
\begin{aligned}
& x \circ y=-\sum_{j=0}^{m} x_{j} y_{j}=\frac{1}{2}(x y+y x)=\operatorname{Scal}[x y] \\
& x \wedge y=\sum_{i<j} e_{i j}\left(x_{i} y_{j}-x_{j} y_{i}\right)=\frac{1}{2}(x y-y x)
\end{aligned}
$$


being a scalar and a two-vector (also called bivector), respectively. In particular, one has that $x^{2}=-|x|^{2}=-\sum_{i=0}^{m} x_{i}^{2}$ and also that $\underline{x}^{2}=-|\underline{x}|^{2}=$ $-\sum_{j=1}^{m} x_{j}^{2}$. Note that for vectors $x$ and $\underline{x}$ we have $\bar{x}=-x$ and $\underline{\bar{x}}=-\underline{x}$.

The Dirac operator in $\mathbb{R}^{0, m+1}$ is the first order vector valued differential operator

$$
\partial_{x}=\sum_{i=0}^{m} e_{i} \partial_{x_{i}}=e_{0} \partial_{x_{0}}+\partial_{\underline{x}}
$$

associated to $x$, where $\partial_{\underline{x}}$ denotes the corresponding Dirac operator in $\mathbb{R}^{0, m}$, associated to $\underline{x}$. Similarly, we may associate the so-called Cauchy-Riemann operator

$$
D=\bar{e}_{0} \partial_{x}=\partial_{x_{0}}+\bar{e}_{0} \partial_{\underline{x}}
$$

and its conjugate $\bar{D}=\partial_{x_{0}}-\bar{e}_{0} \partial_{\underline{x}}$, to the new variable $z$. With respect to any of these respective operators, a notion of monogenicity may be defined. In $\mathbb{R}^{m+1}$ we consider functions taking values in $\mathbb{R}_{0, m+1}$. We say that such a function $f$ is left monogenic in the open region $\Omega$ of $\mathbb{R}^{m+1}$ if and only if $f$ is continuously differentiable in $\Omega$ and satisfies in $\Omega$ the equation $\partial_{x} f=0$, or equivalently, the equation $D f=0$. For an account of the theory of monogenic functions we refer the reader to e.g. $[5,7]$. Observe however that $\Delta_{m}=-\partial_{\underline{x}}^{2}$ and $\Delta_{m+1}=-\partial^{2}=D \bar{D}$, where $\Delta_{m}$ and $\Delta_{m+1}$ denote the respective Laplace operators in $\mathbb{R}^{m}$ and in $\mathbb{R}^{m+1}$. In these factorizations lies the origin of the statement that monogenic functions constitute a refinement of harmonic ones.

In what follows we will also need the Euler operators in $\mathbb{R}^{0, m}$ and $\mathbb{R}^{0, m+1}$, which are respectively given by

$$
E_{m}=-\underline{x} \circ \partial_{\underline{x}}=-\operatorname{Scal}\left(\underline{x} \partial_{\underline{x}}\right)=\sum_{j=1}^{m} x_{j} \partial_{x_{j}}
$$

and

$$
E_{m+1}=-x \circ \partial_{x}=-\operatorname{Scal}\left(x \partial_{x}\right)=\sum_{i=0}^{m} x_{i} \partial_{x_{i}}=x_{0} \partial_{x_{0}}+E_{m}
$$

\section{Polyharmonic functions: some basics}

A function $h^{(p)} \in C_{2 p}(\Omega)$ is called polyharmonic of finite degree $p$, or $(p)$ polyharmonic for short, in the open region $\Omega \subset \mathbb{R}^{m+1}$ if and only if $\Delta^{p} h^{(p)}=0$ in $\Omega$. It is well-known that polyharmonic functions are real-analytic. There is, quite naturally, an extensive literature on the theory of polyharmonic functions, a good reference being [2]. Polyharmonic, and in particular biharmonic $(p=2)$, functions are indeed essential in the study of boundary value problems of mathematical physics, especially from elasticity theory. Let us recall some of their basic properties.

Proposition 1. If the function $h^{(p)}$ is $(p)$-polyharmonic in $\Omega$, then $x_{0}^{j} h^{(p)}$ is $(p+j)$-polyharmonic in $\Omega$. 
Proof. (by induction)

It is easily seen that the commutator $\left[\Delta, x_{0}\right]$ equals $2 \partial_{x_{0}}$. It is also clear that $\partial_{x_{0}} h^{(p)}$ is $(p)$-polyharmonic in $\Omega$. First we prove that $x_{0} h^{(p)}$ is $(p+1)-$ polyharmonic. Indeed, we consecutively have in $\Omega$ :

$$
\begin{aligned}
\Delta^{p+1}\left(x_{0} h^{(p)}\right) & =\Delta^{p}\left(\Delta x_{0} h^{(p)}\right)=\Delta^{p}\left(x_{0} \Delta h^{(p)}+2 \partial_{x_{0}} h^{(p)}\right) \\
& =\Delta^{p}\left(x_{0} \Delta h^{(p)}\right)=\Delta^{p-1}\left(\Delta\left(x_{0} \Delta h^{(p)}\right)\right) \\
& \left.=\Delta^{(p-1}\right)\left(\left(x_{0} \Delta+2 \partial_{x_{0}}\right) \Delta h^{(p)}\right)=\Delta^{p-1} x_{0} \Delta^{2} h^{(p)} \\
& =\ldots=\Delta x_{0} \Delta^{p} h^{(p)}=0
\end{aligned}
$$

Now assume that $x_{0}^{j-1} h^{(p)}$ is $(p+j-1)$-polyharmonic in $\Omega$, then the following computation holds:

$$
\begin{aligned}
\Delta^{p+j}\left(x_{0}^{j} h^{(p)}\right) & =\Delta^{p+j-1}\left(\Delta\left(x_{0} x_{0}^{j-1} h^{(p)}\right)\right) \\
& =\Delta^{p+j-1}\left(x_{0} \Delta+2 \partial_{x_{0}}\right)\left(x_{0}^{j-1} h^{(p)}\right) \\
& =\Delta^{p+j-1}\left(x_{0} \Delta\left(x_{0}^{j-1} h^{(p)}\right)\right) \\
& =\cdots=\Delta x_{0} \Delta^{p+j-1}\left(x_{0}^{j-1} h^{(p)}\right)=0
\end{aligned}
$$

from which the result follows by induction.

Proposition 2. (see also [2])

If the function $h^{(p)}$ is $(p)$-polyharmonic in $\Omega$, then $E_{m+1} h^{(p)}$ also is $(p)$ polyharmonic in $\Omega$, where $E_{m+1}$ is the Euler operator ( 7 ) in $R^{0, m+1}$.

\section{Proof.}

The fundamental commutator here is

$$
\left[\Delta, E_{m+1}\right]=2 \Delta
$$

We then consecutively have

$$
\begin{aligned}
\Delta^{p}\left(E_{m+1} h^{(p)}\right) & =\Delta^{(p-1)}\left(E_{m+1} \Delta+2 \Delta\right) h^{(p)} \\
& =\Delta^{p-1} E_{m+1} \Delta h^{(p)}+2 \Delta^{p} h^{(p)} \\
& =\Delta^{p-2}\left(E_{m+1} \Delta+2 \Delta\right) \Delta h^{(p)}=\Delta^{p-2} E_{m+1} \Delta^{2} h^{(p)} \\
& =\ldots=E_{m+1} \Delta^{p} h^{(p)}=0
\end{aligned}
$$

yielding the statement.

Proposition 3. (see also [2])

If the function $h^{(p)}$ is $(p)$-polyharmonic in $\Omega$, then $\rho^{2 k} h^{(p)}$ also is $(p+k)$ polyharmonic in $\Omega$, where $\rho^{2}$ denotes $|x|^{2}$.

\section{Proof.}

The fundamental commutator here is

$$
\left[\Delta, \rho^{2}\right]=4 E_{m+1}+2(m+1)
$$


First we prove that $\rho^{2} h^{(p)}$ is $(p+1)$-polyharmonic in $\Omega$. We have

$$
\Delta^{p+1}\left(\rho^{2} h^{(p)}\right)=\Delta^{p}\left(\left(\rho^{2} \Delta+4 E_{m+1}+2(m+1)\right) h^{(p)}\right)
$$

which, in view of Proposition 2, reduces to $\Delta^{p} \rho^{2} \Delta h^{(p)}$. Proceeding in the same way, we arrive eventually at $\Delta \rho^{2} \Delta^{p} h^{(p)}$, which is zero indeed. Assume now that $\rho^{2 k-2} h^{(p)}$ is $(p+k-1)$-polyharmonic in $\Omega$. Then we consecutively have:

$$
\begin{aligned}
\Delta^{p+k}\left(\rho^{2 k} h^{(p)}\right) & =\Delta^{p+k-1} \Delta \rho^{2}\left(\rho^{2(k-1)} h^{(p)}\right) \\
& =\Delta^{p+k-1}\left(\rho^{2} \Delta+4 E_{m+1}+2(m+1)\right)\left(\rho^{2 k-2} h^{(p)}\right) \\
& =\Delta^{p+k-1} \rho^{2} \Delta\left(\rho^{2 k-2} h^{(p)}\right) \\
& =\ldots=\Delta \rho^{2} \Delta^{p+k-1}\left(\rho^{2 k-2} h^{(p)}\right)=0
\end{aligned}
$$

which proves the statement.

For the sake of completeness let us mention the following Almansi type decomposition theorem; for a proof we refer to [2].

Theorem 1. If the function $h^{(p)}$ is $(p)$-polyharmonic in a star shaped region $\Omega$ centred at the origin, then there exist unique functions $h_{0}, h_{1}, \ldots, h_{p-1}$, each harmonic in $\Omega$, such that in $\Omega$ holds:

$$
h^{(p)}=h_{0}+\rho^{2} h_{1}+\rho^{4} h_{2}+\ldots+\rho^{2 p-2} h_{p-1}
$$

\section{Biharmonic functions: the Goursat decomposition}

The Goursat decomposition formula for biharmonic functions in Euclidean space is obtained through a series of lemmata.

Lemma 1. Given a real-valued harmonic function $h$ on $\mathbb{R}^{m+1}$, there exists a real-valued harmonic function $h_{0}$ on $\mathbb{R}^{m+1}$ such that $h=\partial_{x_{0}} h_{0}$.

\section{Proof.}

Take an arbitrary point $x_{0}^{*}$ on the $x_{0}$-axis and put

$$
h_{0}\left(x_{0}, \underline{x}\right)=\int_{x_{0}^{*}}^{x_{0}} h(t, \underline{x}) d t+\alpha(\underline{x})
$$

with $\alpha(\underline{x})$ a real-valued smooth function on $\mathbb{R}^{m+1}$. It is clear that $\partial_{x_{0}} h_{0}=h$. In order for the function $h_{0}$ to be harmonic, the function $\alpha(\underline{x})$ should satisfy

$$
\Delta h_{0}=\partial_{x_{0}} h_{0}-\int_{x_{0}^{*}}^{x_{0}} \partial_{\underline{x}}^{2} h(t, \underline{x}) d t-\partial_{\underline{x}}^{2} \alpha=0
$$

Since $h$ is supposed to be harmonic on the whole of $\mathbb{R}^{m+1}$, it holds that $\partial_{\underline{x}}^{2} h=\partial_{x_{0}}^{2} h$, which turns (8) into

$$
\Delta_{m} \alpha(\underline{x})=-\partial_{x_{0}} h\left(x_{0}^{*}, \underline{x}\right)
$$


with $\Delta_{m}$ the Laplace operator in $\mathbb{R}^{m}$. This equation (9) indeed is solvable for $\alpha(\underline{x})$ due to the well-known surjectivity of the Laplace operator $\Delta_{m}$ on $C_{\infty}\left(\mathbb{R}^{m}\right)$.

Remark 1. With the notations of Lemma 1, consider the function

$$
H=h-\overline{e_{0}} \partial_{\underline{x}} h_{0}
$$

and compute

$$
\begin{aligned}
D H & =\left(\partial_{x_{0}}+\overline{e_{0}} \partial_{\underline{x}}\right)\left(h-\overline{e_{0}} \partial_{\underline{x}} h_{0}\right) \\
& =\partial_{x_{0}} h-\overline{e_{0}} \partial_{\underline{x}} \partial_{x_{0}} h_{0}+\overline{e_{0}} \partial_{\underline{x}} h-\overline{e_{0}} \partial_{\underline{x}} \overline{e_{0}} \partial_{\underline{x}} h_{0} \\
& =\partial_{x_{0}} h-\overline{e_{0}} \partial_{\underline{x}} h+\overline{e_{0}} \partial_{\underline{x}} h+\Delta_{m} h_{0} \\
& =\left(\partial_{x_{0}}^{2}+\Delta_{m}\right) h_{0}=\Delta_{m+1} h_{0}=0 .
\end{aligned}
$$

This means that the function $H$ is monogenic in $\mathbb{R}^{m+1}$, which, in its turn, implies that $\left(-\overline{e_{0}} \partial_{\underline{x}} h_{0}\right)$ is a conjugate harmonic function to $h$ in $\mathbb{R}^{m+1}$ in the sense of [6]. Similarly it is shown that $\overline{D H}=0$, in other words: the function $\bar{H}$ is anti-monogenic in $\mathbb{R}^{m+1}$. Moreover

$$
\bar{D} h_{0}=\left(\partial_{x_{0}}-\overline{e_{0}} \partial_{\underline{x}}\right) h_{0}=\partial_{x_{0}} h_{0}-\overline{e_{0}} \partial_{\underline{x}} h_{0}=H
$$

which means that the function $h_{0}$ is a harmonic $\bar{D}$-primitive or potential of the monogenic function $H$. Evenso $D h_{0}=\bar{H}$ implying that $h_{0}$ is a harmonic $D$-primitive of the anti-monogenic function $\bar{H}$.

The above considerations immediately lead to the following results.

Lemma 2. Given a real-valued harmonic function $h$ in $\mathbb{R}^{m+1}$, there exists a monogenic function $H$ in $\mathbb{R}^{m+1}$ with values in $\mathbb{R} \oplus \overline{e_{0}} \mathbb{R}_{m}^{(1)}$, such that $h=$ $\operatorname{Scal}(H)=\operatorname{Scal}(\bar{H})$.

Lemma 3. Given a real-valued harmonic function $h$ in $\mathbb{R}^{m+1}$, there exist monogenic functions $G$ and $H$ in $\mathbb{R}^{m+1}$ with values in $\mathbb{R} \oplus \overline{e_{0}} \mathbb{R}_{m}^{(1)}$, such that

$$
x_{0} h=\frac{1}{2} \operatorname{Scal}[G+\bar{z} H]=\frac{1}{2} \operatorname{Scal}[\bar{G}+z \bar{H}]
$$

with $h=\operatorname{Scal}(H)=\operatorname{Scal}(\bar{H})$.

\section{Proof.}

By Lemma 2 we know the existence of the monogenic function $H=h-$ $\overline{e_{0}} \partial_{\underline{x}} h_{0}$, with values in $\mathbb{R} \oplus \overline{e_{0}} \mathbb{R}_{m}^{(1)}$, the scalar part of which is precisely the given harmonic function $h$. Let us compute

$$
\bar{z} H=x_{0} h-x_{0} \overline{e_{0}} \partial_{\underline{x}} h_{0}-\overline{e_{0}} \underline{x} h+\underline{x} \partial_{\underline{x}} h_{0}
$$

and

$$
z \bar{H}=x_{0} h+x_{0} \overline{e_{0}} \partial_{\underline{x}} h_{0}+\overline{e_{0}} \underline{x} h-\underline{x} \partial_{\underline{x}} h_{0}
$$

from which it follows that

$$
\operatorname{Scal}[\bar{z} H]=x_{0} h-E_{m} h_{0}=\operatorname{Scal}[z \bar{H}]
$$


Taking into account that

$$
E_{m} h_{0}=\left(E_{m+1}-x_{0} \partial_{x_{0}}\right) h_{0}=E_{m+1} h_{0}-x_{0} h
$$

we eventually find that

$$
\text { Scal }[\bar{z} H]=2 x_{0} h-E_{m+1} h_{0}=\operatorname{Scal}[z \bar{H}]
$$

By Proposition 2 we know that $E_{m+1} h_{0}$ is a (real-valued) harmonic function, and hence, by Lemma 2, there exists a monogenic function $G$ in $\mathbb{R}^{m+1}$, with values in $\mathbb{R} \oplus \overline{e_{0}} \mathbb{R}_{m}^{(1)}$, such that

$$
\frac{1}{2} E_{m+1} h_{0}=\frac{1}{2} \operatorname{Scal}[G]=\frac{1}{2} \operatorname{Scal}[\bar{G}]
$$

It then follows that

$$
\begin{aligned}
x_{0} h & =\frac{1}{2} \operatorname{Scal}[\bar{z} H]+\frac{1}{2} E_{m+1} h_{0}=\frac{1}{2} \operatorname{Scal}[z \bar{H}]+\frac{1}{2} E_{m+1} h_{0} \\
& =\frac{1}{2} \operatorname{Scal}[\bar{z} H+G]=\frac{1}{2} \operatorname{Scal}[z \bar{H}+\bar{G}]
\end{aligned}
$$

Lemma 4. Given a real-valued biharmonic function $h^{(2)}$ in $\mathbb{R}^{m+1}$, there exist real-valued harmonic functions $g$ and $h$ in $\mathbb{R}^{m+1}$ such that

$$
h^{(2)}=g+x_{0} h
$$

\section{Proof.}

Clearly the function $\Delta h^{(2)}$ is harmonic in $\mathbb{R}^{m+1}$, so, in virtue of Lemma 1 , there ought to exist a real-valued harmonic function $h_{0}$ in $\mathbb{R}^{m+1}$ such that $\partial_{x_{0}} h_{0}=\Delta h^{(2)}$. Then

$$
\Delta\left(x_{0} h_{0}\right)=\left(x_{0} \Delta+2 \partial_{x_{0}}\right) h_{0}=2 \Delta h^{(2)}
$$

which implies that $h^{(2)}-\frac{1}{2} x_{0} h_{0}$ is a harmonic function, say $g$, in $\mathbb{R}^{m+1}$. It follows that

$$
h^{(2)}=g+x_{0} \frac{h_{0}}{2}=g+x_{0} h
$$

with $g$ and $h$ real-valued harmonic functions in $\mathbb{R}^{m+1}$.

Lemma 5. (Goursat for biharmonic functions)

Given the real-valued biharmonic function $h^{(2)}$, there exist monogenic functions $G$ and $H$ in $\mathbb{R}^{m+1}$ with values in $\mathbb{R} \oplus \overline{e_{0}} \mathbb{R}_{m}^{(1)}$, such that

$$
h^{(2)}=\operatorname{Scal}[G+\bar{z} H]=\operatorname{Scal}[\bar{G}+z \bar{H}]
$$

\section{Proof.}

By Lemma 4 there exist real-valued harmonic functions $g$ and $h$ such that $h^{(2)}=g+x_{0} h$ in $\mathbb{R}^{m+1}$. By Lemma 2 there exists a monogenic function $G_{1}$ in $\mathbb{R}^{m+1}$, with values in $\mathbb{R} \oplus \overline{e_{0}} \mathbb{R}_{m}^{(1)}$, such that

$$
g=\operatorname{Scal}\left[G_{1}\right]=\operatorname{Scal}\left[\overline{G_{1}}\right]
$$


By Lemma 3 there exist monogenic functions $G_{2}$ and $H$, with values in $\mathbb{R} \oplus$ $\overline{e_{0}} \mathbb{R}_{m}^{(1)}$, such that

$$
x_{0} h=\operatorname{Scal}\left[z \bar{H}+\overline{G_{2}}\right]=\operatorname{Scal}\left[\bar{z} H+G_{2}\right]
$$

with $h=2 \operatorname{Scal}[H]=2 \operatorname{Scal}[\bar{H}]$. It follows that

$$
h^{(2)}=\operatorname{Scal}\left[G_{1}\right]+\operatorname{Scal}\left[G_{2}+\bar{z} H\right]=\operatorname{Scal}[G+\bar{z} H]
$$

or

$$
h^{(2)}=\operatorname{Scal}\left[\overline{G_{1}}\right]+\operatorname{Scal}\left[\overline{G_{2}}+z \bar{H}\right]=\operatorname{Scal}[\bar{G}+z \bar{H}]
$$

with $H$ and $G=G_{1}+G_{2}$ monogenic functions in $\mathbb{R}^{m+1}$ with values in $\mathbb{R} \oplus \overline{e_{0}} \mathbb{R}_{m}^{(1)}$.

\section{Polyharmonic functions: the Goursat representation}

Inspired by the case of biharmonic functions in the foregoing section, we proceed similarly, through a series of lemmata, to obtaining the Goursat representation formula for polyharmonic funtions.

Lemma 6. Given a real-valued $(p)$-polyharmonic function $h^{(p)}$ in $\mathbb{R}^{m+1}$, there exist real-valued harmonic functions $g, h_{1}, \ldots, h_{p-1}$ in $\mathbb{R}^{m+1}$, such that

$$
h^{(p)}=g+x_{0} h_{1}+x_{0}^{2} h_{2}+\ldots+x_{0}^{p-1} h_{p-1}
$$

Proof. (by induction)

Recall that, by Lemma 4 , the statement holds for $p=2$. Now we assume that the result is valid for a $(p-1)$-polyharmonic function in $\mathbb{R}^{m+1}$. Clearly the function $\Delta h^{(p)}$ is $(p-1)$-polyharmonic in $\mathbb{R}^{m+1}$. So, by the induction hypothesis, there exist real-valued harmonic functions $g^{\prime}, h_{1}^{\prime}, \ldots, h_{p-2}^{\prime}$ in $\mathbb{R}^{m+1}$ such that

$$
\Delta h^{(p)}=g^{\prime}+x_{0} h_{1}^{\prime}+x_{0}^{2} h_{2}^{\prime}+\ldots+x_{0}^{p-2} h_{p-2}^{\prime}
$$

Since the function $h_{p-2}^{\prime}$ is harmonic in $\mathbb{R}^{m+1}$, there exists, in view of Lemma 1 , a real-valued harmonic function $h_{p-2,0}^{\prime}$ such that $\partial_{x_{0}} h_{p-2,0}^{\prime}=h_{p-2}^{\prime}$. It then follows, by a straightforward computation, that

$$
\Delta\left(\frac{1}{2(p-1)} x_{0}^{p-1} h_{p-2,0}^{\prime}\right)=\frac{p-2}{2} x_{0}^{p-3} h_{p-2,0}^{\prime}+x_{0}^{p-2} h_{p-2}^{\prime}
$$

and hence

$$
\Delta h^{(p)}=g^{\prime}+x_{0} h_{1}^{\prime}+\ldots+x_{0}^{p-3} h_{p-3}^{\prime \prime}+\Delta\left(x_{0}^{p-1} h_{p-1}\right)
$$

where we have put

$$
h_{p-3}^{\prime \prime}=h_{p-3}^{\prime}-\frac{p-2}{2} h_{p-2,0}^{\prime}
$$

and

$$
h_{p-1}=\frac{1}{2(p-1)} h_{p-2,0}^{\prime}
$$


Since $h_{p-3}^{\prime \prime}$ is a real-valued harmonic function in $\mathbb{R}^{m+1}$, there exists, again by Lemma 1 , a real-valued harmonic function $h_{p-3,0}^{\prime \prime}$, such that $\partial_{x_{0}} h_{p-3,0}^{\prime \prime}=$ $h_{p-3}^{\prime \prime}$. It then follows that

$$
\Delta\left(\frac{1}{2(p-2)} x_{0}^{p-2} h_{p-3,0}^{\prime \prime}\right)=\frac{p-3}{2} x_{0}^{p-4} h_{p-3,0}^{\prime \prime}+x_{0}^{p-3} h_{p-3}^{\prime \prime}
$$

and hence

$$
\Delta h^{(p)}=g^{\prime}+x_{0} h_{1}^{\prime}+\ldots+x_{0}^{p-4} h_{p-4}^{\prime \prime}+\Delta\left(x_{0}^{p-2} h_{p-2}\right)+\Delta\left(x_{0}^{p-1} h_{p-1}\right)
$$

where we have put

$$
h_{p-4}^{\prime \prime}=h_{p-4}^{\prime}-\frac{p-3}{2} h_{p-3,0}^{\prime \prime}
$$

and

$$
h_{p-2}=\frac{1}{2(p-2)} h_{p-3,0}^{\prime \prime}
$$

Proceeding in a similar way we eventually arrive at

$$
\Delta h^{(p)}=g^{\prime}+x_{0} h_{1}^{\prime \prime}+\Delta\left(x_{0}^{3} h_{3}+\ldots+x_{0}^{p-2} h_{p-2}+x_{0}^{p-1} h_{p-1}\right)
$$

with real-valued harmonic functions $h_{1}^{\prime \prime}, h_{3}, h_{4}, \ldots, h_{p-2}, h_{p-1}$ in $\mathbb{R}^{m+1}$. Since $h_{1}^{\prime \prime}$ is a real-valued harmonic function in $\mathbb{R}^{m+1}$, there exists, once more by Lemma 1 , a real-valued harmonic function $h_{1,0}^{\prime \prime}$ in $\mathbb{R}^{m+1}$ such that $\partial_{x_{0}} h_{1,0}^{\prime \prime}=$ $h_{1}^{\prime \prime}$ and for which

$$
\Delta\left(\frac{1}{4} x_{0}^{2} h_{1,0}^{\prime \prime}\right)=x_{0} h_{1}^{\prime \prime}+\frac{1}{2} h_{1,0}^{\prime \prime}
$$

leading to

$$
\Delta h^{(p)}=g^{\prime \prime}+\Delta\left(x_{0}^{2} h_{2}+x_{0}^{3} h_{3}+\ldots+x_{0}^{p-1}\right)
$$

with $g^{\prime \prime}$ and $h_{2}$ real-valued harmonic functions in $\mathbb{R}^{m+1}$. Finally, and again by Lemma 1 , there exists a real-valued harmonic function $g_{0}^{\prime \prime}$ in $\mathbb{R}^{m+1}$ such that $\partial_{x_{0}} g_{0}^{\prime \prime}=g^{\prime \prime}$, and for which

$$
\Delta\left(x_{0} g_{0}^{\prime \prime}\right)=2 g^{\prime \prime}
$$

leading to

$$
\Delta h^{(p)}=\Delta\left(x_{0} h_{1}+x_{0}^{2} h_{2}+\ldots+x_{0}^{p-1} h_{p-1}\right)
$$

where we have put $h_{1}=\frac{1}{2} g_{0}^{\prime \prime}$. This implies that the function

$$
h^{(p)}-\left(x_{0} h_{1}+\ldots+x_{0}^{p-1} h_{p-1}\right)
$$

is a real-valued harmonic function, say $g$, in $\mathbb{R}^{m+1}$, from which the desired result follows.

Lemma 7. For each $j \in \mathbb{N}$ one has, for the monogenic function $H$ of Lemma 2, that

$$
\text { Scal }\left[z^{j} \bar{H}\right]=\operatorname{Scal}\left[\bar{z}^{j} H\right]=2^{j} x_{0}^{j} h+g^{(j)}
$$

with $h=\operatorname{Scal}[H]=\operatorname{Scal}[\bar{H}]$ and $g^{(j)}$ a real-valued $(j)$-polyharmonic function in $\mathbb{R}^{(m+1)}$. 


\section{Proof.}

First note that the result in the case where $j=1$, already has been obtained in the proof of Lemma 3. For the sake of simplicity we introduce the notations

$$
\alpha=\overline{e_{0}} \underline{x} \quad \text { and } \quad \beta=\overline{e_{0}} \partial_{\underline{x}}
$$

and we have

$$
\alpha^{2}=-|\underline{x}|^{2}=x_{0}^{2}-\rho^{2}
$$

and also

$$
\operatorname{Scal}[\alpha \beta]=\operatorname{Scal}\left[\underline{x} \partial_{\underline{x}}\right]=\underline{x} \circ \partial_{\underline{x}}=-E_{m}
$$

Now we assume that $j$ is even, say $j=2 k$, and we compute

$$
\begin{aligned}
z^{2 k}= & \left(x_{0}+\alpha\right)^{2 k}=\sum_{i=0}^{2 k}\left(\begin{array}{c}
2 k \\
i
\end{array}\right) x_{0}^{2 k-i} \alpha^{i} \\
= & x_{0}^{2 k}+\left(\begin{array}{c}
2 k \\
2
\end{array}\right) x_{0}^{2 k-2} \alpha^{2}+\ldots+\left(\begin{array}{c}
2 k \\
2 k-2
\end{array}\right) x_{0}^{2} \alpha^{2 k-2}+\left(\begin{array}{c}
2 k \\
2 k
\end{array}\right) \alpha^{2 k} \\
& \quad+\left(\begin{array}{c}
2 k \\
1
\end{array}\right) x_{0}^{2 k-1} \alpha+\left(\begin{array}{c}
2 k \\
3
\end{array}\right) x_{0}^{2 k-3} \alpha^{3}+\ldots+\left(\begin{array}{c}
2 k \\
2 k-1
\end{array}\right) x_{0} \alpha^{2 k-1} \\
= & x_{0}^{2 k}+\left(\begin{array}{c}
2 k \\
2
\end{array}\right) x_{0}^{2 k-2}\left(x_{0}^{2}-\rho^{2}\right)+\ldots+\left(\begin{array}{c}
2 k \\
2 k-2
\end{array}\right) x_{0}^{2}\left(x_{0}^{2}-\rho^{2}\right)^{k-1}+\left(\begin{array}{c}
2 k \\
2 k
\end{array}\right)\left(x_{0}^{2}-\rho^{2}\right)^{k} \\
& \left.+\left(\begin{array}{c}
2 k \\
1
\end{array}\right) x_{0}^{2 k-1}+\left(\begin{array}{c}
2 k \\
3
\end{array}\right) x_{0}^{2 k-3}\left(x_{0}^{2}-\rho^{2}\right)+\ldots+\left(\begin{array}{c}
2 k \\
2 k-1
\end{array}\right) x_{0}\left(x_{0}^{2}-\rho^{2}\right)^{k-1}\right) \alpha \\
= & 2^{2 k-1} x_{0}^{2 k}+\left(a_{1} x_{0}^{2 k-2} \rho^{2}+a_{2} x_{0}^{2 k-4} \rho^{4}+\ldots+a_{k} \rho^{2 k}\right) \\
& +\left(2^{2 k-1} x_{0}^{2 k-1}+b_{1} x_{0}^{2 k-3} \rho^{2}+\ldots+b_{k-1} x_{0} \rho^{2 k-2}\right) \alpha
\end{aligned}
$$

where all coefficients $a_{1}, \ldots, a_{k}, b_{1}, \ldots, b_{k-1}$ are natural numbers. Next we compute, using the monogenic function $H=h-\overline{e_{0}} \partial_{\underline{x}} h_{0}$ of Lemma 2,

Scal $\left[z^{2 k} \bar{H}\right]=\operatorname{Scal}\left[z^{2 k}\left(h+\beta h_{0}\right)\right]$

$$
\begin{aligned}
= & 2^{2 k-1} x_{0}^{2 k} h+\left(a_{1} x_{0}^{2 k-2} \rho^{2}+a_{2} x_{0}^{2 k-4} \rho^{4}+\ldots+a_{k} \rho^{2 k}\right) h \\
& \quad+\left(2^{2 k-1} x_{0}^{2 k-1}+b_{1} x_{0}^{2 k-3} \rho^{2}+\ldots+b_{k-1} x_{0} \rho^{2 k-2}\right)\left(-E_{m}\right) h_{0} \\
= & 2^{2 k} x_{0}^{2 k}+\left(\left(a_{1}+b_{1}\right) x_{0}^{2 k-2} \rho^{2}+\ldots+\left(a_{k-1}+b_{k-1}\right) x_{0}^{2} \rho^{2 k-2}+a_{k} \rho^{2 k}\right) h \\
& \quad-\left(2^{2 k-1} x_{0}^{2 k-1}+b_{1} x_{0}^{2 k-3} \rho^{2}+\ldots+b_{k-1} x_{0} \rho^{2 k-2}\right) E_{m+1} h_{0} \\
= & 2^{2 k} x_{0}^{2 k} h+g^{(2 k)}
\end{aligned}
$$

where $g^{(2 k)}$ is a real-valued $(2 k)$-polyharmonic function in $\mathbb{R}^{m+1}$ in virtue of the Propositions 1,2 and 3 .

The case where $j$ is odd proceeds along similar lines. Also the computations for Scal $\left[\bar{z}^{j} H\right]$ are similar.

Proposition 4. (Goursat for polyharmonic functions)

Given the real-valued $(p)$-polyharmonic function $h^{(p)}$ in $\mathbb{R}^{m+1}$, there exist monogenic functions $H_{0}, H_{1}, \ldots, H_{p-1}$ in $\mathbb{R}^{m+1}$, with values in $\mathbb{R} \oplus \overline{e_{0}} \mathbb{R}_{m}^{(1)}$, such that

$$
\begin{aligned}
h^{(p)} & =\operatorname{Scal}\left(H_{0}+\bar{z} H_{1}+\bar{z}^{2} H_{2}+\ldots+\bar{z}^{p-1} H_{p-1}\right] \\
& =\operatorname{Scal}\left(\overline{H_{0}}+z \overline{H_{1}}+z^{2} \overline{H_{2}}+\ldots+z^{p-1} \overline{H_{p-1}}\right]
\end{aligned}
$$


Proof. (by induction)

We assume that the stated Goursat representation formula is valid for $(p-1)-$ polyharmonic functions in $\mathbb{R}^{m+1}$. Note that the result for $p=2$ has been obtained in Lemma 5. By Lemma 6 we know the existence of real-valued harmonic functions $g, h_{1}, h_{2}, \ldots, h_{p-1}$ in $\mathbb{R}^{m+1}$ such that

$$
h^{(p)}=g+x_{0} h_{1}+x_{0}^{2} h_{2}+\ldots+x_{0}^{p-1} h_{p-1}
$$

or

$$
h^{(p)}=g_{1}^{(p-1)}+x_{0}^{p-1} h_{p-1}
$$

where $g_{1}^{(p-1)}=g+x_{0} h_{1}+x_{0}^{2} h_{2}+\ldots+x_{0}^{p-2} h_{p-2}$ clearly is a $(p-1)$ polyharmonic function in $\mathbb{R}^{m+1}$. Since $h_{p-1}$ is a real-valued harmonic function in $\mathbb{R}^{m+1}$, there exists, in view of Lemma 2 , a monogenic function

$$
H_{p-1}=h_{p-1}-\overline{e_{0}} \partial_{\underline{x}} h_{p-1,0}
$$

with values in $\mathbb{R} \oplus \overline{e_{0}} \mathbb{R}_{m}^{(1)}$, such that $h_{p-1}=\operatorname{Scal}\left[H_{p-1}\right]=\operatorname{Scal}\left[\overline{H_{p-1}}\right]$, and, by Lemma 7 , we know that

$$
\text { Scal }\left[\bar{z}^{p-1} H_{p-1}\right]=\operatorname{Scal}\left[z^{p-1} \overline{H_{p-1}}\right]=2^{p-1} x_{0}^{p-1} h_{p-1}+g_{2}^{(p-1)}
$$

with $g_{2}^{(p-1)}$ also a $(p-1)$-polyharmonic function in $\mathbb{R}^{m+1}$. It follows that

$$
h^{(p)}=g_{1}^{(p-1)}+\frac{1}{2^{p-1}} \operatorname{Scal}\left[\bar{z}^{p-1} H_{p-1}\right]-\frac{1}{2^{p-1}} g_{2}^{(p-1)}
$$

or

$$
h^{(p)}=g^{(p-1)}+\text { Scal }\left[\bar{z}^{p-1} \frac{1}{2^{p-1}} H_{p-1}\right]
$$

with $g^{(p-1)}=g_{1}^{(p-1)}-\frac{1}{2^{p-1}} g_{2}^{(p-1)}$ a $(p-1)$-polyharmonic function in $\mathbb{R}^{m+1}$, for which, by the induction hypothesis, the proposition holds. So there exist monogenic functions $H_{0}, H_{1}, \ldots, H_{p-2}$ in $\mathbb{R}^{m+1}$, with values in $\mathbb{R} \oplus \overline{e_{0}} \mathbb{R}_{m}^{(1)}$, such that

and the result follows.

$$
g_{1}^{(p-1)}=\operatorname{Scal}\left[H_{0}+\bar{z} H_{1}+\ldots+\bar{z}^{p-2} H_{p-2}\right]
$$

\section{References}

[1] E. Almansi, Sull' integrazione dell' equazione differenziale $\Delta^{2 m} u=0$, Ann. Mat. Pura Appl., 3(2) (1899), pp. 1-51.

[2] N. Aronszajn, T.M. Creese, L.J. Lipkin, Polyharmonic functions, Clarendon Press, Oxford (1983).

[3] S. Bock and K. Gürlebeck, On a spatial generalization of the KolosovMuskhelishvili formulae, Math. Methods Appl. Sci., 32(2) (2009), pp. 223-240.

[4] F.A. Bogashov, Representation of a biharmonic function in the complex space $\mathbb{C}^{2}$, Russian Acad. Sci. Dokl. Math., 48(2) (1994), pp. 259-262.

[5] F. Brackx, R. Delanghe and F. Sommen, Clifford Analysis, Pitman Publishers, Boston-London-Melbourne (1982).

[6] F. Brackx, R. Delanghe and F. Sommen, On Conjugate Harmonic Functions in Euclidean Space, Math. Methods Appl. Sci., 25 (2002), 1553-1562. 
[7] R. Delanghe, F. Sommen and V. Souček, Clifford Algebra and Spinor-Valued Functions, Kluwer Academic Publishers, Dordrecht (1992).

[8] E. Fischer, Über die Differentiationsprozesse der Algebra, J. für Math., 148 (1917), pp. 1-78.

[9] Ph. Frank and R.V. Mises (eds.), Die Differential- und Integralgleichungen der Mechanik und Physik, vol. 1, Dover, N-Y (1961).

[10] E. Goursat, Sur l'équation $\Delta \Delta u=0$, Bull. Soc. Math. France, 26 (1898), pp. $236-237$.

[11] M. Krakowski and A. Charnes, Stokes' paradox and biharmonic flows, Report 37, Carnegie Institute of Technology, Department of Mathematics, Pittsburgh, PA (1953).

Fred Brackx

Clifford Research Group, Faculty of Engineering and Architecture, Ghent University

Galglaan 2, 9000 Gent, Belgium

e-mail: fb@cage.ugent.be

Richard Delanghe

Clifford Research Group, Faculty of Engineering and Architecture, Ghent University

Galglaan 2, 9000 Gent, Belgium

e-mail: rd@cage ugent. be

Hennie De Schepper

Clifford Research Group, Faculty of Engineering and Architecture, Ghent University

Galglaan 2, 9000 Gent, Belgium

e-mail: hds@cage . ugent . be

Vladimir Souček

Mathematical Institute, Faculty of Mathematics and Physics, Charles University Prague

Sokolovska 83, Prague, Czech Republic

e-mail: soucek@karlin.mff.cuni.cz 